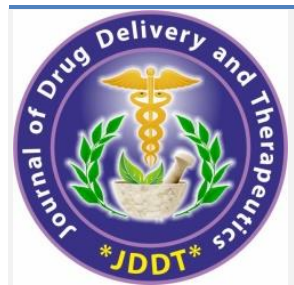

Open Access Full Text Article

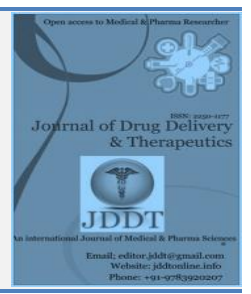

\title{
Will Mesenchymal Stem Cell Therapy Be Effective In COVID-19?
}

\author{
Srinivas K (D), Senthil M*(D), R Sambath Kumar (D), R Kameshwaran \\ Department of Pharmacy Practice, Department of Pharmaceutics, J.K.K.Nattraja College of Pharmacy, Kumarapalayam-638183, Tamil Nadu, India
}

\section{Article Info:}

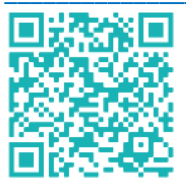

Article History:

Received 23 September 2021 Reviewed 26 October 2021 Accepted 04 November 2021 Published 15 November 2021

\section{Cite this article as:}

Srinivas K, Senthil M, Sambath Kumar R Kameshwaran R, Will Mesenchymal Stem Cell Therapy Be Effective In COVID-19?, Journal of Drug Delivery and Therapeutics. 2021; 11(6):281-285

DOI: http://dx.doi.org/10.22270/jddt.v11i6.5143

\section{*Address for Correspondence:}

Senthil M, Department of Pharmacy Practice, Department of Pharmaceutics, J.K.K.Nattraja College of Pharmacy, Kumarapalayam-638183, Tamil Nadu, India

ORCID ID: https://orcid.org/0000-0002-8741-362X

\section{Abstract}

Background: The World Health Organization (WHO) reports that the outbreak of the deadly virus had been noted almost in all the countries worldwide. Newly no standard therapies are available to combat the situation and this remains the major challenge for healthcare professionals to provide effective treatment against the life-threatening condition. A potential regenerative medicine method using the infusion of stem cells for the treatment of lung disorders has been reported. This review attempted to explore the immunomodulatory characteristics of Mesenchymal Stem Cells (MSCs) and how these properties make them beneficial for the treatment of Severe Acute Respiratory Syndrome coronavirus 2 (SARS-CoV2) patients.

Objectives: To study the effect of Mesenchymal Stem Cell therapy in treating COVID-19.

Methodology: A literature search was conducted to identify recent research relating to the review's goal of analyzing the relevance of stem cells in battling SARS-CoV-2.

Results: The MSCs settle in the lungs intravenously to enhance the pulmonary microenvironment, minimize immune system over-activation, and encourage regeneration of damaged lung tissues. Its therapeutic properties like immune response inhibition play a major role in combating viruses. The avoidance of cytokine storm is the most important stage in COVID-19 therapy. Their potent immunomodulatory properties have positive effects in avoiding or attenuating the cytokine storm and assisting in the regeneration of injured lung tissues/other organs.

Conclusion: Intravenous human Umbilical Cord-Mesenchymal Stem Cell therapy (hUC-MSC) transplantation is a safe and effective technique that may be used as a restoration and prioritized therapeutic option for treating severe COVID-19.

Keywords: Covid-19, human Umbilical Cord-Mesenchymal Stem Cell therapy (huc-msc), Immune system.

\section{INTRODUCTION}

In late December 2019, the first cases of pneumonia with an unknown origin were reported in Wuhan, China ${ }^{1}$. The pathogen was identified as a novel coronavirus that has a similar molecular structure to SARS-CoV-2 ${ }^{2}$. Since then, not only in China but throughout the world, the number of COVID-19 patients has risen dramatically. Coronaviruses (CoVs) are part of the Orthocoronavirinae subfamily of the Coronaviridae family of the Order Nidovirales. The Orthocoronavirinae subfamily is subdivided into four genera those includes Alphacoronavirus (-CoV), Betacoronavirus ($\mathrm{CoV})$, Gammacoronavirus (-CoV), and Deltacoronavirus ($\mathrm{CoV}){ }^{3,4}$. The World Health Organization reports that the outbreak of the deadly virus had been noted almost in all the countries worldwide. Newly no standard therapies are available to combat the situation and this remains the major challenge for healthcare professionals to provide effective treatment against the life-threatening condition. As a result, concerted efforts are required to develop safe and effective COVID-19 therapies, particularly for severe instances. A potential regenerative medicine method using the infusion of stem cells for the treatment of lung disorders has been reported ${ }^{5-7}$. The recent study finds that infusing stem cells in the affected individuals showed promising results as the stem cells with their cytoprotective and pro-antigenic properties remain as an advanced treatment strategy in severe lung diseases to overcome this pandemic period 8-11. This review attempted to explore the immunomodulatory characteristics of MSCs and how these properties make them beneficial for the treatment of SARS-CoV-2 patients.

\section{METHODOLOGY}

A literature search was conducted via online search through PubMed, Embase, and various resources with the help of keywords to identify recent research relating to the review's goal of analyzing the relevance of stem cells in battling SARSCoV-2.

\section{RESULTS AND DISCUSSION}

\section{NEW TREATMENT APPROACH INVOLVED IN COVID-19}

\subsection{Nanotechnology-Based Treatment Techniques}

COVID-19 treatments rely heavily on nanotechnology ${ }^{12}$. The effectiveness of nanotechnology in SARS CoV-2 treatments is 
dependent on selecting the correct nanocarriers for the right medication candidate ${ }^{13}$. Furthermore, nanocarriers circumvent the limits of current antiviral treatments. In COVID-19, nanoparticle-assisted regulation of antigenpresenting cells (APCs) is critical for vaccine development ${ }^{14}$. Even though antimicrobial medicines such as chloroquine, redeliver, and lopinavir had shown promising results against SARS CoV-2, some patients had severe adverse effects ${ }^{15}$. The association of nano-carriers offers the essential environment for these medicines to work without adverse effects ${ }^{16}$. During the early phases of COVID-19, nano-macrophage mimetic systems neutralize viral activity and, afterward, decrease inflammation ${ }^{17}$. The consequences of COVID-19 hematological pathology can be mitigated by NanoErythrocyte mimic medication delivery ${ }^{18}$. Combination medication treatments are important in the treatment of COVID-19 because of their low side effects, low dose amount, and multiple targeting. Nanocarriers have been identified as promising candidates for multi-drug delivery, which is beneficial for combination medication therapy ${ }^{19}$. Nanoparticle antigen delivery to dendritic cells enhances $\mathrm{T}$ cell immunity 20. The use of nanomaterials such as Nanospheres, nanocarriers, liposomes, lipid nanoparticles, nanopages, and dendrimers can improve targeted medication delivery for the treatment of COVID-19 21. In addition to nanotechnology, stem cell therapy plays an important role in the treatment of COVID-19 illness.

\subsection{Stem cell therapy and various methods of stem cell therapy}

The term "stem cell" refers to a wide range of different cell types. They are either completely undifferentiated or partially differentiated cells that may proliferate continuously to generate more of the same kind of cell and can develop into a variety of cell types. The modifiers "embryonic" and "adult" are commonly used to distinguish stem cells based on the developmental stage of the animal from which they originate, but these terms are becoming obsolete as new research has discovered how to convert fully differentiated adult cells back into embryonic stem cells and, conversely, adult stem cells, more correctly termed "somatic" stem cells meaning "from the body", back into embryonic stem cells 22. Adult stem cells such as hematopoietic stem cells (MSCs) from adipose tissues have an excellent capacity to repair tissues because they can proliferate for longer periods while remaining undifferentiated and then differentiate into various types of cells. These cells are more commonly used in therapeutic procedures due to several ethical and regulatory constraints 23, 24. MSCs' immunomodulatory properties have been reported to be one of the major elements imparting therapeutic benefits during the process of lung repair and regeneration in a variety of pathological conditions including bronchopulmonary dysplasia, asthma, acute lung injury, chronic obstructive pulmonary disease, and idiopathic pulmonary fibrosis. Several attempts are being made in this direction $25-27$.

\subsection{Outlook of mesenchymal stem cell therapy}

MSCs can regenerate or renew cells on their own and they are differentiated by several lineages. MSCs can also be isolated from various adult tissues, such as bone marrow (BM), peripheral blood (PB), and adipose tissues (AT) (such as abdominal fat, infrapatellar fat pad, and buccal fat pad), as well as neonatal birth-associated tissues, such as placenta (PL), umbilical cord (UC), Warton jelly (WJ), amniotic fluid (AF), and cord blood (CB), and then stored for future. As a result, it appears that MSC-based therapy, or at least a mix of treatments, might be an attractive option for clinical trials in
COVID-19 patients 28, 29, 30. This is because the simplicity with which these sources may be extracted and the amount collected make them ideal for both experimental and therapeutic uses. Many MSCs have recently been generated from novel sources, including menstrual blood and endometrium 31,32. Bone marrow, adipose tissue, umbilical cord blood, and endothelial progenitor cells are the most popular types of MSCs used for lung disease treatment 33,34 . Despite tremendous progress in the field of stem cell-based treatment, the primary constraints of this therapeutic strategy immunogenicity, restricted cell supplies, and ethical issues, have yet to be resolved. MSCs have gotten a lot of interest because of their source potential, high proliferation rate, less invasive technique, and lack of ethical concerns when compared to other therapies they have a significant advantage ${ }^{35}$. COVID-19 may trigger a destroying immune overreaction in the body and it produces large amounts of inflammatory factors, causing a cytokine storm including, an overproduction of immune cells and cytokines ${ }^{36}$.

\subsection{Mechanism of MSCs}

T-cells are activated during inflammation through several cell signaling processes. The initial signal that activates lymphocytes is mediated by antigen-specific $\mathrm{T}$ cell receptors (TCR), whereas the second phase, known as co-stimulation, is independent of the TCR yet critical for allowing complete immune response activation and avoiding energy ${ }^{37}$. MSCs can repress T-cells at both the primary and secondary activation phases by signalling through soluble substances such as cytokines and growth factors, as well as through mechanisms involving direct cell-to-cell interactions ${ }^{38}$. IFNpromotes indole amine 2, 3-dioxygenase (IDO) activity in MSCs via the JAK/STAT signaling pathway 39, 40, which converts tryptophan to kynurenine, which inhibits many cells and reduces the inflammatory response. Apoptosis of pro-inflammatory T-cells was found after increasing IDO activity by tryptophan deprivation due to a generalized decrease in cellular energetics, as evidenced by increased production and accumulation of released kynurenines ${ }^{41}$.

Surprisingly, more than 30 soluble molecules have been found to promote MSC immunomodulation potential to control T-cell activation and proliferation. Human MSCs, in contrast to other species, generate IDO to inhibit T-cell proliferation 42 and induce $\mathrm{T}$ cell energy, which is characterized by a lack of proliferation and a reduction in cytokine output ${ }^{43}$. These findings highlight the fact that MSCs have the greatest immune-inhibitory effect on T-cell proliferation ${ }^{38}$. Cell-to-cell interactions are also important in MSC-mediated immunomodulation. MSCs do not express costimulatory molecules such as CD40, CD80, CD86, CD134, and CD252 even after being exposed to inflammatory signals 44, 45. Furthermore, MSCs can inhibit T-cell proliferation by expressing CD39 and producing adenosine, which activates the adenosine A2 receptor (ADORA2A) on the surface of lymphocytes ${ }^{46}$. It is worth noting that MSCs from diverse origins do not have the equivalent immunoregulatory ability.

In research comparing MSCs from various sources, Warton Jelly produced MSCs shown to be the most efficient in immunosuppression ${ }^{45}$. According to research, activation of Toll-like receptors (TLRs) plays a critical role in immunomodulation mediated by cell-to-cell interaction as well as MSC-secreted soluble substances. TLRs have been discovered to have a crucial function in the innate immune system for recognizing pathogen-associated molecular patterns (PAMPs) and activating initial responses against pathogens ${ }^{47}$. It is important to note that MSCs from different sources do not have the same immunoregulatory capacity. TLR3 and TLR4 ligation has been shown to boost the 
immunosuppressive characteristics of MSCs in the absence of IFN- by enhancing tryptophan breakdown, which leads to increased kynurenine synthesis, which is known to be catalyzed by IDO and triggered by autocrine interferon 48 . MSCs may be polarised into two homogeneous phenotypes by downstream TLR signaling, known as MSC1 and MSC2. Low-level TLR4 agonist exposure polarises MSC1 toward pro-inflammatory activity via processes that are not completely understood. In contrast, for phenotype 2, MSCs can be polarised by TLR3 activation, and as a result, MSCs inhibit the immune response ${ }^{49}$. To minimize negative effects while utilizing MSCs as an anti-inflammatory treatment, it has been proposed to activate particular TLRs under culture conditions before using the cells in vivo ${ }^{50}$. Human leukocyte antigen (HLA) class I molecules are expressed at low levels in MSCs. This characteristic of stem cells in general aids them in evading natural killer (NK) cells. Furthermore, MSCs and other stem cells lack the expression of HLA class II molecules as well as costimulatory molecules such as CD40, CD40L, CD80, and CD86, which are important in the activation of T-lymphocyte-mediated immunological responses 6, 42, 43, 51. MSCs with "immune privileged" properties, in addition to being no immunogenic, that is, do not elicit a rejection response due to less strong proinflammatory IFN-induced HLA-II expression, making them an appealing therapeutic tool for treating COVID-19 52.

\subsection{Mesenchymal stem cell therapy treating other viral infectious diseases}

When compared to highly differentiated cells, MSCs are usually resistant to viral infections. This ability is provided by the presence of IFN-stimulated genes (ISG), which can target at many stages of the viral cycle, preventing viruses from crossing the cell membrane, blocking the endocytic route, mRNA transcription, nuclear import of mRNAs, genome integration/amplification, protein translation, virus assembly and release 36, 53,54. PMAIP1, ISG15, IFI6, IFITM, SAT1, p21/CDKN1A, SERPINE1, and CCL2 are among the ISGs expressed in MSCs 36,54 . These ISG work by inhibiting several viral infections in vitro, including dengue, Ebola, SARS, and influenza-A 53. Wu and colleagues (2018) demonstrated the significance of certain MSC-derived-ISGs by silencing p21/CDKN1A expression, which resulted in enhanced MSC susceptibility to chikungunya virus infection. 74 Stem Cell Rev and Rep (2021) 17:71-93 IFITM3 silencing, however, made MSCs more vulnerable to yellow fever and zika virus infection 36, 55. They discovered that IDO expressing MSC stimulated with IFN- in vitro decreased HIV$1 / 2$ virion production. The authors hypothesized that this impact was caused by tryptophan deficiency, which inhibits the production of emerging viral proteins 56 .

MSCs have been shown in pre-clinical trials to be helpful in a variety of viral infections ${ }^{57}$. Chan and colleagues found that influenza A/H5N1 infection reduced alveolar fluid clearance (AFC) and increased alveolar protein permeability (APP) in human alveolar epithelial cells (AEC) in vitro, both of which are linked with acute lung damage 58. Human BM-MSCs inhibited or decreased this impact, which was mediated in part by angiopoietin-1 (Ang1) and keratinocyte growth factor (KGF) production in the co-culture experiment. In elderly A/H5N1-infected mice, MSC therapy on day 5 postinfection enhanced survival and body weight, as well as improved lung pathology and histopathology scores 57 .

\subsection{The mechanism behind mesenchymal stem cell therapy treating covid-19}

China, the United States, Jordan, Iran, and numerous other countries have recently launched cell-based treatment clinical investigations, and some findings have been published (Table 1). Research on a female patient with acute COVID19 syndrome was published in China, and the findings of laboratory tests and CT scans revealed highly successful outcomes after 21 days of therapy with umbilical cord MSCs. A recent case study of a case report of a 65-year-old female patient diagnosed with COVID-19 in critical condition revealed the precise $2019 \mathrm{nCoV}$ variant now known as SARSCoV-2 59 .

The unfortunate SARS-CoV-2 outbreak has imposed an urgent demand from scientists and clinicians worldwide to find effective therapeutic agents to alleviate sufferings in COVID-19 patients on the one hand, while also enforcing a demand for effective vaccines to mitigate its spread in the future and spared or unaffected present populations on the other. Cell therapy and gene therapy are currently advanced disciplines of research used to treat a wide range of illnesses. It is important to note that the inflammatory cytokine profiles generated by H5N1 and COVID-19 are comparable 60 , including exceptionally high amounts of IL-6, GCSF, IP10, MCP-1, MIP1A, and TNF-, which are likely to cause serious organ damage and mortality in large numbers 61,62 . MSC recipients had considerably lower levels of TNF-, a powerful pro-inflammatory cytokine, and higher levels of IL-10, an anti-inflammatory cytokine, as compared to placebo control individuals. When administered intravenously, a portion of the MSCs settle in the lungs as well, which may enhance the pulmonary microenvironment and, as a result, minimize immune system over-activation and encourage regeneration of damaged lung tissues 63,64 .

Cytokine dysregulation has also been linked to influenza pathogenesis produced by a variety of influenza A virus subtypes. The most prevalent etiology found in these viral infection situations is pneumonia and acute respiratory distress syndrome. Intravenous injection of MSCs to patients suffering from influenza and other pulmonary illnesses has shown promising results in restoring lung functions and correcting the lung functional and structural losses caused by the infection's cytokine storm. As a result, it is reasonable to think that MSC treatment can benefit COVID-19 patients via immunomodulatory pathways 65. Its therapeutic properties like immune response inhibition play a major role in combating viruses. Their capacity to develop into type II alveolar epithelial (AT2) cells in vitro may also aid ${ }^{66}$. Lung damage may change the signals of the BMP4-NFATc1-TSP1 signaling pathway, resulting in abnormal alveolar progenitor function. TSP1, a substance produced by MSCs, may help to re-establish damaged vascular signals and promote epithelial healing ${ }^{67}$. As a result, avoidance of cytokine storm is seen to be the most important stage in COVID-19 therapy. MSCs, due to their potent immunomodulatory properties, may have positive effects in avoiding or attenuating the cytokine storm and assisting in the regeneration of injured lung tissues/other organs.

\section{CONCLUSION}

The infectious viral SARS-CoV-2 produces COVID-19, which predominantly affects the respiratory system and lungs, resulting in pneumonia and organ damage. These microorganisms induce a strong immunological response, resulting in excessive cytokine production, which might be referred to as a "cytokine storm. "Genetic tests revealed that this virus is closely linked to SARS-CoV and is most likely to be found in wild bats. Because of the constant interaction with wildlife in markets, the outbreak was likely triggered by a zoonotic process.Even though the pandemic just began a few months ago, much genomic and phylogenetic information on the causal culprit has already been published. SARS-CoV-2, like other previously discovered 
coronaviruses, infects host cells by attaching to the ACE2 receptor, and it is closely related to bat-SL-CoVZC45 and batSL-CoVZXC21, both of which have been detected in wild bat populations 68 .

HUC-MSC therapy, as a non-invasive treatment, is a very successful and promising way for clinical use and promotion at this critical time due to the absence of viable methods to treat severe COVID-19 69. However, the cost-effectiveness and timeliness of therapeutic preparation are the most capable discussed topics for MSC-based therapy for COVID19 , but human life is more valuable, and COVID-19 is so dangerous. As a result, clinical trials of MSC treatment to treat COVID-19 are still some time away, however, there are some intriguing results to consider. Stem cell therapy, particularly MSCs, maybe one of the most effective therapies, or a combination of treatments, for COVID-19 patients. However, scientists are working tirelessly to create a COVID19 vaccine as well as medicines to treat this condition.

\section{Conflicts of Interest: No conflicts of interest.}

Funding statement: This review did not receive any funding.

\section{REFERENCES}

1. Guan WJ, Ni ZY, Hu Y, et al. Clinical characteristics of coronavirus disease 2019 in China. N Engl J Med. 2020; 382:1708-20.10.doi:1056/NEJMoa2002032

2. Lu R, Zhao X, Li J, et al. Genomic characterisation and epidemiology of 2019 novel coronavirus: implications for virus origins and receptor binding. Lancet. 2020; 395:565-74. https://doi.org/10.1016/ S0140-6736(20)30251-8

3. Banerjee A, Kulcsar K, Misra V, et al. Bats and coronaviruses. Viruses. 2019; 11(1):41.doi:10.3390/v11010041

4. Yang D, Leibowitz JL.The structure and functions of coronavirus genomic 3' and 5' ends. Virus Res. 2015; 206:120-33. doi:10.1016/j.virusres.2015.02.025

5. Kotton, DN. Next-generation regeneration. American Journal of Respiratory and Critical Care Medicine.2012; 185(12):12551260.

doi:https://www.atsjournals.org/doi/full/10.1164/rccm.20120 2-0228PP

6. Weiss DJ. Concise review: Current status of stem cells and regenerative medicine in lung biology and diseases. Stem Cells.2014; 32(1):16-25.doi:10.1002/stem.1506

7. Fernanda F, Patricia RM. Stem-Cell extracellular vesicles and lung repair. Stem Cell. 2017; 4:1-11. doi:10.21037/sci.2017.09.02

8. Li F, Li W, Farzan M, et al. Structural biology: Structure of SARS coronavirus spike receptor-binding domain complexed with receptor. Science. 2005; 309(5742):1864-1868.doi: https://doi.org/10.1126/science.1116480

9. World Health Organization. WHO Director-General's opening remarks at the media briefing on COVID-19 - 11 March 2020. Retrieved March 14, 2020. Available at: https://www.who.int/dg/speeches/detail/ who-directorgeneral-s-opening-remarks-at-the-media-briefing-oncovid19\%2D\%2D-11-march-2020

10. Ji Y, Ma Z, Peppelenbosch MP, et al. Potential association between COVID-19 mortality and health-care resource availability. The Lancet Glob. 2020; 8(4):e480. doi: https://doi.org/ 10.1016/S2214-109X(20)30068-1

11. Baud D, Qi X, Nielsen-Saines K, et al. Real estimates of mortality following COVID19 infection. The Lancet Infect Dis. 2020; 20(7):773. doi: https://doi.org/ 10.1016/S14733099(20)30195-X

12. Petros RA, DeSimone, JM. Strategies in the design of nanoparticles for therapeutic applications. Nat. Rev. Drug Discov. 2010; 9: 615-627. doi:10.1038/nrd2591

13. Chauhan G, Madou M. J, Kalra S, et al. Nanotechnology for COVID-19: therapeutics and vaccine research. ACS Nano. 2020; 14 (7): 7760-7782. doi:10.1021/acsnano.0c04006

14. Banchereau J, Steinman, R. M. Dendritic cells and the control of immunity. Nature. 1998; 392:245-252. doi:10.1038/32588
15. Mainardes RM, Diedrich C. The potential role of nanomedicine on COVID-19 therapeutics. Therap. Deliv.2020; 11:7-9. doi:10.4155/tde-2020-0069

16. Campos ER, Pereira AE, de Oliveira JL, et al. How can nanotechnology help to combat COVID-19? opportunities and urgent need. J. Nanobiotechnol. 2020; 18: 1-23. doi:10.1186/s12951-020-00685-4

17. Zhang Q, Honko A, Zhou J, et al. Cellular nanosponges inhibit SARS-CoV-2 infectivity. Nano Lett. 2020; 20: 5570-5574. doi:10.1021/acs.nanolett.0c02278

18. Cavezzi A., Troiani E, Corrao S. COVID-19: hemoglobin, iron, and hypoxia beyond inflammation. A narrative review. Clin. Pract. 2020; 10: 1271. doi:10. 4081/cp.2020.1271

19. Destache CJ, Belgum T, Christensen K, et al. Combination antiretroviral drugs in PLGA nanoparticle for HIV-1. BMC Infect. Dis. 2009; 9: 198. doi:10.1186/1471-2334-9-198

20. Joffre OP, Segura E, Savina A, et al. Cross-presentation by dendritic cells. Nat. Rev. Immunol.2012; 12: 557-569. doi:10.1038/nri3254

21. Witika BA, Makoni PA, Mweetwa LL, et al. Nano-biomimetic drug delivery vehicles: potential approaches for COVID-19 treatment. Molecules. 2020; 25: 5952. doi:10.3390/molecules 25245952

22. Bajada S, Mazakova I, Richardson JB, et al. Updates on stem cells and their application in regenerative medicine. J Tissue Eng Regen Med. 2008; 2(4):169-83. doi:10.1002/term.83

23. Lee KD, Kuo TK, Whang-Peng J, et al. In vitro hepatic differentiation of human mesenchymal stem cells. Hepatology. 2004; 40(6):1275-1284. doi: 10.1002/hep.20469

24. Ratajczak MZ, Marycz K, Poniewierska-Baran A, et al. Very small embryonic-like stem cells as a novel developmental concept and the hierarchy of the stem cell compartment. Adv Med Sci. 2014; 59(2):273-280. doi: 10.1016/j.advms.2014.08.001

25. Yang J, Jia Z. Cell-based therapy in lung regenerative medicine. Regenerative Medicine Research. 2014; 2:1-7. doi: 10.1186/2050-490X-2-7

26. Behnke J, Kremer S, Shahzad T,et al. MSC based therapies-New perspectives for the injured lung. J Clin Med. 2020; 3(9):682. doi: $10.3390 / \mathrm{jcm} 9030682$

27. Weiss DJ. Mesenchymal stem cells for lung repair and regeneration. Stem cells in the respiratory system (pp. 25-42). New York: Springer Science \& Business Media.

28. Ding DC, Shyu WC, Lin SZ, et al.Current con-cepts in adult stem cell therapy for stroke. Curr. Med. Chem. 2006;13(29):35653574. doi: $10.2174 / 092986706779026237$

29. Ding DC, Shyu WC, Lin SZ, et al. The role of endothelial progenitor cells in ischemic cerebral and heart diseases. Cell $\begin{array}{llll}\text { Transplant. 2007; 16(3):273-284. doi: } & \end{array}$ $10.3727 / 00000000778346477$

30. Potten CS, Loeffler M. Stem cells: Attributes, cycles, spirals, pitfalls and uncertainties. Lessons for and from the crypt. Development. 1990; 110(4):1001-1020. PMID: 2100251

31. Dennis JE, Carbillet JP, Caplan AI, et al. The STRO-1+ marrow cell population is multipotential. Cells Tissues Organs. 2002; 170(23):73-82. doi: 10.1159/000046182

32. Torensma R, Jansen JA, Figdor CG. Ceramic hydroxyapatite coating on titanium implants drives selective bone marrow stromal cell adhesion. Clin. Oral Implants Res. 2003; 14(5):569577. doi: 10.1034/j.1600-0501.2003.00949.x

33. Gronthos S, Graves SE, Ohta SJ, et al. The STRO-1+ fraction of adult human bone marrow contains the osteogenic precursors. Blood.1994; 84(12):4164-4173. PMID: 7994030

34. Gronthos S, Zannettino AC, Hay SJ, et al. Molecular and cellular characterisation of highly purified stromal stem cells derived from human bone marrow. J. Cell Sci. 2003; 116(9):1827-1835. doi: $10.1242 /$ jcs. 00369

35. Golchin A, Farahany, TZ, Khojasteh A, et al. The clinical trials of Mesenchymal stem cell therapy in skin diseases: An update and concise review. Curr Stem Cell Res Ther. 2019; 14(1), 22-33. https://doi.org/10. 2174/1574888x13666180913123424

36. Khoury M, Cuenca J, Cruz FF, et al. Current status of cell-based therapies for respiratory virus infections: Applicability to COVID-19. Eur Respir J. 2020; 55(6): 2000858. doi: https://doi.org/10. 1183/13993003.00858-2020

37. Frauwirth KA, Thompson CB. Activation and inhibition of lymphocytes by Costimulation. J Clin Invest. 2002; 109: 295299. doi: $10.1172 / J C I 14941$ 
38. Glennie S, Soeiro I, Dyson PJ, et al. Bone marrow Mesenchymal stem cells induce division arrest anergy of activated $\mathrm{T}$ cells. Blood. 2005; 105: 2821-2827. doi: 10.1182/blood-2004-093696

39. Rawlings JS, Rosler KM, Harrison DA. The JAK/ STAT signaling pathway. J Cell Sci. 2004; 117: 1281- 1283. doi: 10.1242/jcs.00963

40. Meisel R, Zibert A, Laryea M, et al. Human bone marrow stromal cells inhibit allogeneic T-cell responses by indoleamine 2,3dioxygenase-mediated tryptophan degradation. Blood. 2004; 103(12):4619-4621. doi: 10.1182/blood-2003-11-3909

41. Mbongue J, Nicholas D, Torrez T, et al. The role of indoleamine 2, 3-dioxygenase in immune suppression and autoimmunity. Vaccines. 2015; 3:703-729. doi: https://doi.org/10.3390/vaccines3030703

42. Haddad R, Saldanha-Araujo F. Mechanisms of T-cell immunosuppression by mesenchymal stromal cells: What do we know so far? Bio Med Research International.2014:1-14. doi: https://doi.org/10.1155/2014/216806

43. Stagg J. Immune regulation by mesenchymal stem cells: Two sides to the coin. Tissue Antigens. 2007; 69(1):1-9. doi 10.1111/j.1399-0039.2006.00739.x

44. Briones J, Novelli S, Sierra J. T-cell costimulatory molecules in acute-graft-versus host disease: Therapeutic implications. Bone Marrow Res. 2011; 2011: 976793. doi: 10.1155/2011/976793

45. Najar M, Raicevic G, Kazan HF, at al. Immune-related antigens, surface molecules and regulatory factors in human-derived mesenchymal stromal cells: The expression and impact of inflammatory priming. Stem Cell Rev Rep. 2012;8(4): 11881198. doi: 10.1007/s12015-012-9408-1

46. Saldanha-Araujo F, Ferreira FIS, Palma PV, et al. Mesenchymal Stromal cells up-regulate CD39 and increase adenosine production to suppress activated T-lymphocytes. Stem Cell Res. 2011; 7(1): 66-74. doi: 10.1016/j.scr.2011.04.001

47. Pevsner-Fischer M, Morad V, Cohen-Sfady M, et al. Toll-like receptors and their ligands control mesenchymal stem cell functions. Blood. 2007; 109(4): 1422-1432. doi: 10.1182/blood-2006-06-028704

48. Opitz CA, Litzenburger UM, Lutz C, et al. Toll-like receptor engagement enhances the immunosuppressive properties of human bone marrow-derived mesenchymal stem cells by inducing indoleamine-2,3-dioxygenase- 1 via interferon- $\beta$ and protein kinase R. Stem Cells. 2009; 27(4): 909-919. doi: $10.1002 /$ stem.7

49. Bunnell BA, Betancourt AM, Sullivan DE. New concepts on the immune modulation mediated by mesenchymal stem cells. Stem Cell Res Ther. 2010; 1(5):34. doi: 10.1186/scrt34

50. Raicevic G, Rouas R, Najar M, et al. Inflammation modifies the pattern and the function of toll-like receptors expressed by human mesenchymal stromal cells. Hum Immunol. 2010; 71(3): 235-244. doi: 10.1016/j.humimm.2009.12.005

51. Rasmusson I. Immune modulation by mesenchymal stem cells. Exp Cell Res. 2006; 312(12): 2169-2179. doi: 10.1016/j.yexcr.2006.03.019

52. Gao F, Chiu SM, Motan DAL, et al. Mesenchymal stem cells and immunomodulation: Current status and future prospects. Cell Death Dis. 2016; 7(1): e2062-e2062. doi: 10.1038/cddis.2015.327

53. Bailey CC, Zhong G, Huang IC, et al. IFITM-family proteins: The Cell's first line of antiviral defense. Annu Rev Virol. 2014; 1(1):261-283. doi: https://doi.org/10. 1146/annurev-virology031413-085537
54. Schoggins JW. Interferon-stimulated genes: What do they all do? Annu Rev Virol. 2019; 6(1): 567-584. doi: https:// doi.org/10.1146/annurev-virology-092818-015756

55. Wu X, Dao Thi VL, Huang Y, et al. Intrinsic Immunity Shapes Viral Resistance of Stem Cells. Cell. 2018; 172(3):423-438.e25 doi: https://doi. org/10.1016/j.cell.2017.11.018

56. Kane M, Zang TM, Rihn SJ, et al. Identification of interferonstimulated genes with antiretroviral activity. Cell Host Microbe. 2016; 20(3): 392-405. doi https://doi.org/10.1016/j.chom.2016.08.005

57. McIntyre LA., Moher D, Fergusson DA, et al. Efficacy of mesenchymal stromal cell therapy for acute lung injury in preclinical animal models: A systematic review. PLoS One. 2016; 11(1). doi: https://doi.org/10.1371/ journal.pone.0147170

58. Chan MCW, Kuok DIT, Leung C, et al. Human mesenchymal stromal cells reduce influenza a H5N1-associated acute lung injury in vitro and in vivo. Proceedings of the National Academy of Sciences of the United States of America. 2016; 113(13): 3621-3626. doi: https://doi.org/10.1073/pnas.1601911113

59. Bing L, Junhui C, Tao L, et al. Clinical remission of a critically ill COVID-19 patient treated by human umbilical cord. ChinaXiv. 2020; 99(31): e21429. doi: https://doi.org/10.12074/ 202002.00084

60. Darwish I, Mubareka S, Liles WC. Immunomodulatory therapy for severe influenza. Expert Rev Anti-infect Ther. 2011; 9: 807822. doi: $10.1586 /$ eri.11.56

61. Huang C, Wang Y, Li X, et al. Clinical features of patients infected with 2019 Novel Coronavirus in Wuhan, China. The Lancet 2020; 395:497-506. doi: 10.1016/S0140-6736(20)30183-5

62. Wang $\mathrm{D}, \mathrm{Hu} \mathrm{B}, \mathrm{Hu} \mathrm{C}$, et al. Clinical characteristics of 138 hospitalized patients with 2019 novel coronavirus- infected pneumonia in Wuhan, China. JAMA. 2020; 323 (11):1061-1069. doi: 10.1001/jama.2020.1585

63. Leng $\mathrm{Z}, \mathrm{Zhu} \mathrm{R}$, Hou $\mathrm{W}$, et al. Transplantation of ACE2mesenchymal stem cells improves the outcome of patients with COVID-19 pneumonia. Aging Dis. 2020; 11(2):216-228. doi 10.14336/AD.2020.0228

64. Shetty AK. Mesenchymal stem cell infusion shows promise for combating coronavirus (COVID-19) induced pneumonia. Aging Dis. 2020; 11(2): 462-464. doi: 10.14336/AD.2020.0301

65. Chen J, Hu C, Chen L, et al. Clinical Study of Mesenchymal Stem Cell Treatment for Acute Respiratory Distress Syndrome Induced by Epidemic Influenza A (H7N9) Infection: A Hint for COVID-19 Treatment. Engineering. 2020; 6(10): 1153-1161 doi: https://doi. org/10.1016/j.eng.2020.02.006

66. Ma N, Gai H, Mei J, et al. Bone marrow mesenchymal stem cells can differentiate into type II alveolar epithelial cells in vitro. Cell Biol Int. 2011; 35(12):1261-1266. doi: 10.1042/CBI20110026

67. Leeman KT, Pessina P, Lee J, et al. Mesenchymal stem cells increase alveolar differentiation in lung progenitor organoid cultures. Sci Rep. 2019; 9: 1-10. doi: 10.1038/s41598-01942819-1

68. Hu D, Zhu C, Ai L, et al. Genomic characterization and infectivity of a novel SARS-like coronavirus in Chinese bats. Emerg Microbes Infect. 2018; 7: 1-10. doi: 10.1038/s41426-018-01555

69. Lei Shu, Changming Niu , Ruyou Li., et al, Treatment of severe COVID-19 with human umbilical cord mesenchymal stem cells. Stem Cell Research \& Therapy. 2020; 11:361. doi: https://doi.org/10.1186/s13287-020-01875-5 